\title{
The Remainder in Linear Methods of Approximation
}

\author{
By W. E. Milne ${ }^{1}$
}

\begin{abstract}
The following discussion grows out of an effort to formulate a systematic treatment of the error for such diverse processes as interpolation, numerical differentiation, numerical integration, harmonic analysis, approximation by Least Squares, approximation by equating moments, and other allied operations.

First of all, a systematic and uniform procedure is exhibited by which a desired method of approximation may be explicitly constructed. Second, by suitable transformation the remainder is put in a form in which it is possible in many cases to estimate its approximate magnitude. And third, the theory is applied to a variety of concrete examples, and bounds are obtained for the magnitude of the error.
\end{abstract}

\section{Fundamental Problem of Approximation}

In the various types of problems to which this investigation applies, we are dealing with a real function $f(x)$ in an interval $a \leqq x \leqq b$, and we are supplied with a more or less adequate description, or definition, of this function. For example, we may have a table of values of $f(x)$ furnished by experimental observations, or a table of values of $f(x)$ computed from a series or an asymptotic expansion, or we may have the values, not of $f(x)$ itself, but of certain of its derivatives, $f^{\prime}(x)$, or $f^{\prime \prime}(x)$ etc. In such instances $f(x)$ is not completely defined by the information provided. On the other hand, $f(x)$ may be a particular solution of a given differential equation, for which no solution in elementary form is known. Or $f(x)$ may be the solution of an integral equation or of some other type of functional equation. In such cases the function $f(x)$ may be completely, though implicitly, defined by the given equation.

The essential point is that we may not have a simple mathematical expression for $f(x)$, and yet we wish to obtain particular values of $f(x)$, or to find its integral, or to find its derivatives - in short to be able to use $f(x)$ just as freely as if it were expressed in terms of known elementary functions.

In order to do these things, a common practice is to replace $f(x)$ by an approximate expression in terms of suitably chosen known functions. How to secure this approximate representation of $f(x)$ is the problem that we shall call the fundamental problem of approximation.

In order that the subsequent analyses can be performed, it will be assumed henceforth that $f(x)$ has a continuous derivative of order $n+1$ in a fixed interval $a \leqq x \leqq b$. The values of $n$ to be selected will appear as we proceed.

\section{Basic Functions}

In all the types of approximation here considered the first essential idea is to employ a basic set of linearly independent known functions $u_{0}(x)$, $u_{1}(x), \ldots, u_{n}(x)$, and to use an appropriately chosen linear combination of these functions as an approximation to the given function. So far as theory is concerned any other set $v_{0}(x), v_{1}(x)$, ..., $v_{n}(x)$, equivalent to the set $\left\{u_{i}(x)\right\}$ by linear combination, might equally well be used. In practical numerical analysis however, it is important to select the set best suited to the numerical process to be employed.

In numerical methods the set of basic functions more often employed than all others put together is the set of polynomials, $1, x, x^{2}, \ldots, x^{n}$. Actually these appear more frequently in various linear combination such as the binomial coefficient functions $\left(\begin{array}{l}x \\ 0\end{array}\right),\left(\begin{array}{l}x \\ 1\end{array}\right), \ldots\left(\begin{array}{l}x \\ n\end{array}\right)$, the Legendre polynomials $P_{0}(x), P_{1}(x), \ldots, P_{n}(x)$, the Lagrangian coefficients $L_{0}^{(n)}(x), L_{1}^{(n)}(x), \ldots L_{n}^{(n)}(x)$, etc.

\footnotetext{
1 Mathématics Department, Oregon State College.
} 
Next in importance probably are the trigonometric functions, $1, \cos x, \sin x, \ldots \cos m x$, $\sin m x$ which may also occur in power form, 1, $\cos x, \sin x, \ldots, \cos ^{m} x, \sin ^{m} x$, or in products of terms such as $\sin \left(x-x_{i}\right) / 2$.

Other sets sometimes used are $e^{-x^{2} / 2} H_{i}(x)$, where $H_{i}(x)$ is an Hermitian polynomial, $e^{-x} L_{i}(x)$, where $L_{\imath}(x)$ is a Laguerre polynomial, etc.

It is assumed henceforth that the basic functions $u_{0}(x), u_{1}(x), . . ., u_{n}(x)$ all possess continuous derivatives of order $n+1$ in the closed interval $a \leqq x \leqq b$, and that the Wronskian determinant

$$
W=\left|\begin{array}{cccc}
u_{0}(x) & u_{1}(x) & \ldots & u_{n}(x) \\
u_{0}^{\prime}(x) & u_{1}^{\prime}(x) & \ldots & u_{n}^{\prime}(x) \\
\ldots & \ldots & \ldots & \ldots \\
u_{0}{ }^{(n)}(x) & u_{1}{ }^{(n)}(x) & \ldots & u_{n}{ }^{(n)}(x)
\end{array}\right|
$$

does not vanish anywhere in the interval $a \leqq x \leqq b$.

\section{Equivalence Operators}

The basic set of approximating functions having been selected, the next step in solving the fundamental problem of approximation is to set up the rule by means of which the coefficients in the approximating linear combination of basic functions are to be determined. We shall consider here only objective, analytic methods, as opposed to subjective and graphical methods. With this restriction, it will be found that in a great many cases of practical importance, the rule is based on what might be called the principle of equivalence. We shall not attempt to formulate a completely general statement of this principle, but shall instead bring out its meaning by citing some familiar examples in which a principle of equivalence is employed. Such examples are:

(a). Equivalence of value at discrete points. That is, the value of the approximating function is made equal to the value of the given function $f(x)$ at $x_{0}, x_{1}, \ldots, x_{n}$.

(b). Equivalence of lineal elements at discrete points. That is, the values of the approximating function and its first derivative are made to agree with $f(x)$ and $f^{\prime}(x)$ at assigned points. This may also be regarded as a limiting case of (a) when pairs of points move into coincidence. (c) Equivalence of weighted definite integrals. That is, the value of

$$
\int_{a}^{b} w_{i}(x) f(x) d x
$$

are made to agree with corresponding expressions formed for the approximating function.

(d) Equivalence of weighted sums. That is, the values of

$$
\sum_{j=0}^{M} w_{i}\left(x_{j}\right) f\left(x_{j}\right)
$$

are made to agree with similar expressions for the approximating function.

Evidently this list can be greatly extended.

We shall henceforth in this paper consider only cases in which the coefficients are completely determined by some appropriate application of the principle of equivalence.

In order to treat all such cases by means of a comprehensive notation, it is convenient to introduce a set of operators $O_{0}, O_{1}, \ldots, O_{n}$, equal in number to the set of basic functions, and called equivalence operators. What these operators actually do in any particular method of approximation is determined by the rule selected for setting up the approximation. For the examples (a), (b), (c), and (d) above the corresponding operators are:

(a) $O_{i} f(x)=f\left(x_{i}\right)$; $i=1, \ldots, n$.

(b) $O_{2 i} f(x)=f\left(x_{i}\right)$, $i=1, \ldots, n$.

$$
O_{2 i+1} f(x)=f^{\prime}\left(x_{i}\right) \text {; }
$$

(c) $O_{i} f(x)=\int_{a}^{b} w_{i}(x) f(x) d x ; \quad i=1, \ldots, n$.

(d) $O_{i} f(x)=\sum_{j=0}^{M} w_{i j} f\left(x_{j}\right)$; $i=1, \ldots, n$.

It is desirable to employ some form of analytical expression for the operators $O_{i}$, sufficiently general to include all types of linear operators likely to arise, but sufficiently restricted to guarantee that subsequent mathematical manipulations can be carried out. Accordingly, it is assumed henceforth that $O_{i} f(x)$ can be expressed by means of Riemann-Stieltjes integrals in the form.

$$
O_{i} f(x)=\sum_{j=0}^{k} \int_{a}^{b} f^{(j)}(x) d q_{i j}(x), k<n,
$$


in which the $q_{i j}(x)$ are suitably chosen functions of bounded variation in the interval $a \leqq x \leqq b$. By specialized choices of the $q_{i j}$ we may obtain each of the particular operators (a), (b), (c), and (d) listed above. For example, if $q_{i t}(x)$ is a. step function with unit step at $x=x_{i}$ while the other $q_{i j}(x)$ vanish we have

$$
O_{i} f(x)=\int_{a}^{b} f^{(t)}(x) d q_{i t}(x)=f^{(t)}\left(x_{i}\right)
$$

Again if $d q_{i 0}(x)=w_{i}(x) d x$ and $q_{i j}=0$ for $j=1$, $2, \ldots, k$, then

$$
O_{i} f(x)=\int_{a}^{b} w_{i}(x) f(x) d x
$$

Also if $q_{i 0}(x)$ is a step function having $M+1$ steps of magnitude $w_{i 0}$ at $x_{0}, w_{i 1}$ at $x_{1}, \ldots, w_{i M}$ at $x_{M}$, and if $q_{i j}=0$, for $j>0$, then

$$
O_{i} f(x)=\sum_{j=0}^{M} w_{i j} f\left(x_{j}\right) .
$$

From the definition of $O_{i}$ as a sum of integrals, it is clear that

(1) The operation $O_{i}$ is distributive with respect to addition, i. e.,

$$
\begin{gathered}
O_{i}\left[f_{1}(x)+f_{2}(x)\right] \equiv O_{i} f_{1}(x)+O_{i} f_{2}(x), \\
\left(O_{1}+O_{2}\right) f(x) \equiv O_{1} f(x)+O_{2} f(x) .
\end{gathered}
$$

(2) If $A$ is constant

$$
O_{i}[A f(x)] \equiv A O_{i} f(x) .
$$

(3) The result of the operation $O_{i}$ on any function $f(x)$ no longer contains $x$ as an independent variable and insofar as the variable $x$ is concerned may be treated as a constant.

\section{The Approximation Formula}

Suppose that the function $f(x)$ has been given in any of the ways suggested in section I, that an appropriate basic set $\left\{u_{i}\right\}$ has been chosen, that a rule for determining the coefficients based on the principle of equivalence has been selected (i. e., the equivalence operators $O_{1}, O_{2}, \ldots, O_{n}$ are decided upon), and that what we desire is a formula expressing the result of some given operation $O$ when performed on $f(x)$, where $O$ satisfies the conditions imposed on the $O_{i}$ in section III. Let the desired approximating linear combination of the $u_{j}$ be

$$
\sum_{j=0}^{n} A_{j} u_{j}(x)
$$

in which the $A$ 's are coefficients to be determined. Then the principle of equivalence gives the $n+1$ equations

$$
O_{i} f(x)=O_{i} \sum_{j=0}^{n} A_{j} u_{j}(x) .
$$

which by the properties stated at the end of section III are the same as

$$
O_{i} f(x)=\sum_{j=0}^{n} A_{\jmath} O_{i} u_{j}(x)
$$

It is assumed henceforth that the determinant

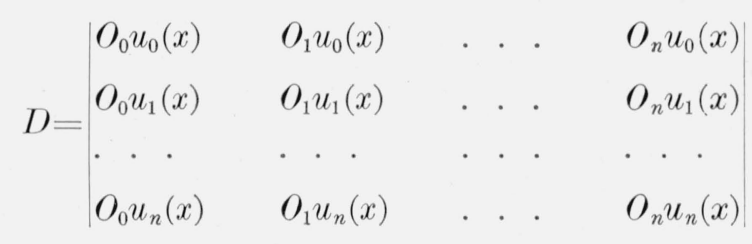

does not vanish. Clearly the $n+1$ eq 1 determine the coefficients $A_{j}$ uniquely.

Our objective is to express the result of the operation $O$ applied to $f(x)$ by means of the same operation, $O$, applied to the approximating function $\Sigma A_{i} u_{i}(x)$, together with a remainder term, $R$, denoting the error of this approximate representation. That is

$$
O f(x)-O \Sigma A_{i} u_{i}(x)=R .
$$

When the values of the $A_{i}$ as determined by eq 1 are substituted in the expression for $R$, the left-hand member may be written in determinant form, and we have the general expression for the error term

$$
\begin{aligned}
& \mid \begin{array}{lllll}
O f(x) & O_{0} f(x) & O_{1} f(x) & \ldots & O_{n} f(x)
\end{array} \\
& O u_{0}(x) \quad O_{0} u_{0}(x) \quad O_{1} u_{0}(x) \backslash \quad \ldots O_{n} u_{0}(x) \\
& \frac{1}{D} O u_{1}(x) \quad O_{0} u_{1}(x) \quad O_{1} u_{1}(x) \quad \ldots O_{n} u_{1}(x)=R \text {. } \\
& \begin{array}{ccccc}
\cdots & \cdots & \cdots & \cdots & \cdots
\end{array}
\end{aligned}
$$

Formula 2 provides the explicit means for setting up any linear approximation formula when the desired operation $O$, the basic set $u_{i}(x)$, and the 
rule for the coefficients based on the principle of equivalence have been selected.

It is obvious from formula 2 that $R$ vanishes whenever $f(x)$ is any linear combination of the basic set $\left\{u_{i}(x)\right\}$. Also, $R$ vanishes if the operator, $O$, is any linear combination of the operators $O_{0}$, $O_{1}, \ldots, O_{n}$.

\section{The Operator $\mathrm{R}$}

Since we have assumed that the operator, $O$, is expressible by means of Riemann-Stieltjes integrals just as are the $O_{i}$, it is clear from formula 2 that $R$ may be looked upon as the result of an operation of this same general type performed on the function $f(x)$ and might be called $O^{\prime} f(x)$. However, we wish to retain the letter $R$ associated with the idea of a remainder term, and hence shall use the notation

$$
R=R[f(x)],
$$

indicating both that $R$ is a remainder and also that $R$ is the result of a linear operator operating on the function $f(x)$.

This operator also obeys the distributive property with respect to addition so that

$$
R\left[f_{1}(x)+f_{2}(x)\right]=R\left[f_{1}(x)\right]+R\left[f_{2}(x)\right] .
$$

Moreover, as we observed at the end of section IV,

$$
R\left[u_{i}(x)\right]=0, \quad i=0, \quad 1, \quad \ldots, \quad n .
$$

These two properties of $R$ are essential in the subsequent developments.

A few examples showing the formation of $R[f(x)]$ in specific cases will be illuminating. In each case $D$ is the cofactor of the element in the upper lefthand corner of the numerator determinant. If the values $x_{0}, x_{1}, \ldots, x_{n}$ are all distinct it may be shown that in none of these cases does $D$ vanish.

Example 1. Polynomial interpolation.

Here the formula is

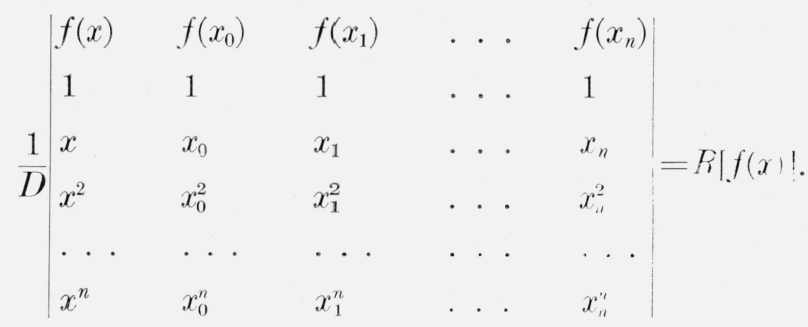

Example 2. Lineal element interpolation.

$\frac{1}{D}\left|\begin{array}{cccccc}f(x) & f\left(x_{0}\right) & f^{\prime}\left(x_{0}\right) & \ldots & f\left(x_{n}\right) & f^{\prime}\left(x_{n}\right) \\ 1 & 1 & 0 & \ldots & 1 & 0 \\ x & x_{0} & 1 & \ldots & x_{n} & 1 \\ x^{2} & x_{0}^{2} & 2 x_{0} & \ldots & x^{2} & 2 x_{n} \\ \ldots & \ldots & \ldots & \ldots & \ldots & \ldots \\ x^{2 n+1} & x_{0}^{2 n+1} & (2 n+1) x_{0}^{2 n} & \ldots & x_{n}^{2 n+1} & (2 n+1) x_{n}^{2 n}\end{array}\right|=R[f(x)]$.

Example 3. Quadrature formula.

$\frac{1}{D}\left|\begin{array}{ccccc}\int_{\alpha}^{\beta} f(x) d x & f\left(x_{0}\right) & f\left(x_{1}\right) & \ldots & f\left(x_{n}\right) \\ \beta-\alpha & 1 & 1 & \ldots & 1 \\ \frac{\beta^{2}-\alpha^{2}}{2} & x_{0} & x_{1} & \ldots & x_{n} \\ \frac{\beta^{3}-\alpha^{3}}{3} & x_{0}^{2} & x_{1}^{2} & \ldots & x_{n}^{2} \\ \ldots & \ldots & \ldots & \ldots & \ldots \\ \frac{\beta^{n+1}-\alpha^{n+1}}{n+1} & x_{0}^{n} & x_{1}^{n} & \ldots & x_{n}^{n}\end{array}\right|=R[f(x)]$.

Journal of Research 
Example 4. Taylor's series with remainder

$\frac{1}{D}\left|\begin{array}{ccccc}f(x) & f\left(x_{0}\right) & f^{\prime}\left(x_{0}\right) & \ldots & \left.f^{(n}\right)\left(x_{0}\right) \\ 1 & 1 & 0 & \ldots & 0 \\ x & x_{0} & 1 & \ldots & 0 \\ x^{2} & x_{0}^{2} & 2 x_{0} & \ldots & 0 \\ \ldots & \ldots & \ldots & \ldots . & \ldots \\ x^{n} & x_{0}^{n} & n x_{0}^{n-1} & \ldots . & n !\end{array}\right|=R[f(x)]$.

Example 5. Least square approximation.

Since any linearly independent set of functions can be made orthonormal by linear combination, the basic set itself may be assumed to be orthonormal with respect to a given interval $a \leqq x \leqq b$. Then with the abridged notation

$$
\int f u=\int_{a}^{b} f(x) u(x) d x, \text { etc., }
$$

the formula is

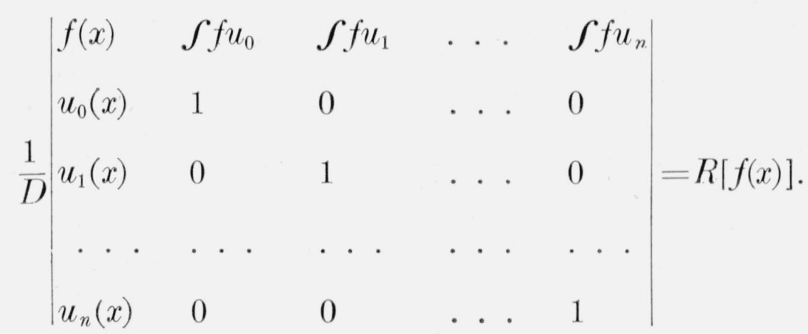

A Fourier series with remainder is a particular case of this example. So also is Least Square approximation using Legendre Polynomials.

The procedure outlined above evidently has great flexibility, since theoretically at least there are infinitely many ways of choosing a basic set of functions, infinitely many ways of selecting the rule for forming the coefficients based on the equivalence principle, and similarly for $f(x)$ and the operator $O$.

It should be emphasized that although eq 2 has been called "an approximation formula", it is not necessarily in any sense a "good" approximation. Only when the remainder term $R[f(x)]$ is small enough to be ignored without affecting the desired accuracy do we call it a good approximation.

\section{The Basic Differential Equation}

Since the functions $u_{i}(x)$ have continuous derivatives of order $n+1$ and the Wronskian, $W$, does not vanish in the interval $a \leqq x \leqq b$, we may construct a homogeneous linear differential equation

$$
\begin{aligned}
& L(u)=D^{n+1} u+P_{1}(x) D^{n} u+ \\
& P_{2}(x) D^{n-1} u+\ldots+P_{n+1}(x) u=0
\end{aligned}
$$

of order $n+1$ with coefficients $P_{i}(x)$ continuous in $a \leqq x \leqq b$, which has the $n+1$ functions $u_{i}(x)$ as a set of linearly independent solutions.

In determinant form this differential equation is

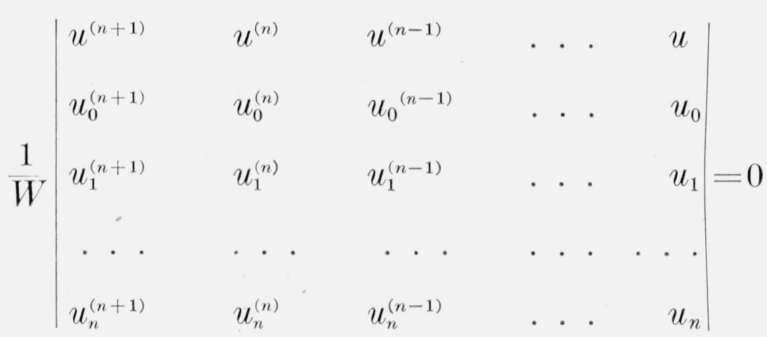

Example 1. For the basic set of polynomials $1, x, \ldots, x^{n}$, the differential equation is simply

$$
L(u)=D^{n+1} u=0 .
$$

Example 2. For the basic trigonometric functions, $1, \cos x, \sin x, \ldots, \cos m x, \sin m x$, the equation is

$$
D\left(D^{2}+1\right)\left(D^{2}+2^{2}\right) \ldots\left(D^{2}+m^{2}\right) u=0 .
$$

Example 3. For the basic hyperbolic set 1, $\cosh x, \sinh x, \ldots, \cosh m x$, sinh $m x$, the equation is

$$
D\left(D^{2}-1\right)\left(D^{2}-2^{2}\right) \ldots\left(D^{2}-m^{2}\right) u=0 .
$$

Example 4. For the basic exponential set $e^{x}, e^{2 x}, \ldots, e^{m x}$ the equation is

$$
(D-1)(D-2) \ldots(D-m) u=0 .
$$


It is of interest to note that in each of these four examples the differential equation has constant coefficients. In general, of course, the coefficients are functions of $x$. It may also be noted that in the first three examples above the differential equation is self-adjoint.

The general solution of the nonhomogeneous differential equation

is

$$
L(u)=\phi(x),
$$

$$
u=\sum_{i=0}^{n} C_{i} u_{i}(x)+\int_{a}^{x} \phi(s) g(x, s) d s,
$$

\begin{tabular}{|c|c|c|c|c|}
\hline & $u_{0}(x)$ & $u_{1}(x)$ & $\ldots$ & $u_{n}(x)$ \\
\hline & $u_{0}^{(n-1)}(s)$ & $u_{1}^{(n-1)}(s)$ & $\ldots$ & $u_{n}^{(n-1)}(s)$ \\
\hline & $\ldots$ & $\ldots$ & $\ldots$ & $\cdots$ \\
\hline & $u_{0}^{\prime}(s)$ & $u_{1}^{\prime}(s)$ & $\ldots$ & $u_{n}^{\prime}(s)$ \\
\hline & $u_{0}(s)$ & $u_{1}(s)$ & $\ldots$ & $u_{n}(s)$ \\
\hline & $u_{0}^{(n)}(s)$ & $u_{1}^{(n)}(s)$ & $\ldots$ & $u_{n}^{(n)}(s)$ \\
\hline & $u_{0}^{(n-1)}(s)$ & $u_{1}^{(n-1)}(s)$ & $\ldots$ & $u_{n}^{(n-1)}(s)$ \\
\hline & $\cdots$ & $\ldots$ & $\cdots$ & $\ldots$ \\
\hline & $u_{0}^{\prime}(s)$ & $u_{1}^{\prime}(s)$ & $\ldots$ & $u_{n}^{\prime}(s)$ \\
\hline & $u_{0}(s)$ & $u_{1}(s)$ & $\ldots$ & $u_{n}(s)$ \\
\hline
\end{tabular}

in which the C's are arbitrary constants and

The essential property of $g(x, s)$ is that

$$
\frac{\partial k}{\partial x^{k}} g(x, s)=\left\{\begin{array}{l}
0 \text { at } a=s \text { for } k=0,1, \ldots, n-1 \\
1 \text { at } x=s \text { for } k=n .
\end{array}\right.
$$

\section{The Function $g(x, s)$}

For the basic set $1, x, x^{2}, \ldots, x^{n}$ we find that

$$
g(x, s)=(x-s)^{n} / n !
$$

For the basic set $1, \cos x, \sin x, \ldots, \cos m x$, $\sin m x$,

$$
g(x, s)=\left[2 \sin \left(\frac{x-s}{2}\right)\right]^{2 n} /(2 n) !
$$

For the set $1, \cosh x, \sinh x, \ldots, \cosh m x$, $\sinh m x$,

$$
g(x, s)=\left[2 \sinh \left(\frac{x-s}{2}\right)\right]^{2 n} /(2 n) !
$$

For the functions $e^{-x^{2} / 2} H_{n}(x)$,

$$
g(x, s)=e^{\left(s^{2}-x^{2}\right) / 2}(x-s)^{n} / n ! .
$$

For the functions $e^{-x} L_{n}(x)$,

$$
g(x, s)=e^{s-x}(x-s)^{n} / n ! .
$$

For the set $e^{x}, e^{2 x}, \ldots, e^{(n+1)} x$,

$$
g(x, s)=\left(e^{x-s}-1\right)^{n} / n ! .
$$

\section{Expression for the Remainder}

Let $f(x)$ be a function with a continuous derivative of order $n+1$ in the region under consideration and set up the nonhomogeneous differential equation

$$
L(u)=L(f(x)) .
$$

A particular solution of this equation is obviously $u=f(x)$, and hence by eq 3

$$
f(x)=\sum a_{i} u_{i}(x)+\int_{a}^{x} L(f(s)) g(x, s) d s .
$$

Suppose now that we have some particular operator, $R$, where $R$ is formed for the same basic set as is eq 6. Applying the operator, $R$, to eq 6 we get, in view of distributive property of $R$,

$$
R[f(x)]=\sum a_{i} R\left[u_{i}(x)\right]+R\left[\int_{a}^{x} L(f(s)) g(x, s) d s\right] .
$$

Since $R\left[u_{i}(x)\right]=0, i=0,1, \ldots, n$, the first term on the right drops out. Now $R$ applied to any function $\varphi(x)$ has the form

$$
R[\varphi(x)]=\sum_{i=0}^{k} \int_{a}^{b} \varphi^{(i)}(x) d q_{i}(x), \quad k<n,
$$

where the $q_{i}(x)$ are of bounded variation in $a \leqq x \leqq b$.

Consequently

$$
\begin{aligned}
& R\left[\int_{a}^{x} L(f(s)) g(x, s) d s\right]= \\
& \sum_{i=0}^{k} \int_{a}^{b} \frac{d^{i}}{d x^{i}}\left[\int_{a}^{x} L(f(s)) g(x, s) d s\right] d q_{i}(x) .
\end{aligned}
$$

By successive differentiations of the integral and use of eq (5) at each step we obtain

$$
\frac{d^{i}}{d x^{i}}\left[\int_{a}^{x} L(f(s)) g(x, s)\right]=\int_{a}^{x} L(f(s)) \frac{\partial^{i}}{\partial x^{i}} g(x, s) d s ;
$$


hence

$$
\begin{gathered}
R\left[\int_{a}^{x} L(f(s)) g(x, s) d s\right] \\
\left.\left.=\sum_{i=0}^{k} \int_{a}^{b} \int_{a}^{x} L\right) f(s)\right) \frac{\partial^{i}}{\partial x^{i}} g(x, s) d s d q_{i}(x) .
\end{gathered}
$$

It is convenient to define $\bar{g}(x, s)$ by the conditions

$$
\bar{g}(x, s)=\left\{\begin{array}{ll}
g(x, s) & \text { for } x \geqq s \\
0 & \text { for } x<s .
\end{array}\right\}
$$

Then the functions $\partial^{i} \bar{g}(x, s) / \partial x^{i}, i=0,1, \ldots, n-1$, are continuous in the square $a \leqq x \leqq b, a \leqq s \leqq b$, and vanish identically in that half of the square for which $x<s$. Hence in the double integral above we may replace $g$ by $\bar{g}$, replace the variable upper limit $x$ by the constant limit $b$, interchange the order of the integrations, and put the summation under the first integral. The result is

$$
R\left[\int_{a}^{x} L(f(s)) g(x, s) d s\right]=\int_{a}^{b} L(f(s)) G(s) d s,
$$

where we have set

$$
\begin{aligned}
G(s) & =\sum_{i=0}^{k} \int_{a}^{b} \frac{\partial^{i}}{\partial x^{j}} \bar{g}(x, s) d q_{i}(x) \\
& =R[\bar{g}(x, s)] .
\end{aligned}
$$

We have therefore

$$
R[f(x)]=\int_{a}^{b} L(f(s)) G(s) d s,
$$

in which

$$
G(s)=R[\bar{g}(x, s)] .
$$

Note that $R$ operates on $\bar{g}(x, s)$ as a function of $x$, not $s$. Equation 8 provides the desired expression for the remainder term. It should be noted that eq 8 is merely a mathematical identity and therefore in one sense tells us nothing new. Actually, however, eq 8 accomplishes the important step of separating the problem into two parts, one of which, $L(f(s))$, is independent of the operator $R$, and the other, $G(s)$, independent of the function $f(x)$.

\section{The Function $G(s)$}

For brevity the function $G(s)$ has been written as a function of the single variable $s$, since $s$ is the variable of immediate concern in the integral of eq 8. In reality $G(s)$ depends, directly or in- directly, also on all the values of $x$, fixed or variable, that are implied in the performance of the operation $R$. When $s$ is larger than the largest $x$ involved in the operation $R[f(x)]$, the function $G(s)$ vanishes identically because for all such values of $s$ the function $\bar{g}(x, s)$ is zero by definition. Again if $s$ is less than the least $x$ involved in $R[f(x)]$ the function $G(s)$ vanishes identically, this time because $\bar{g}(x, s)=g(x, s)$ and is therefore simply a linear combination of the $u_{i}(x)$, so that

$$
R[\bar{g}(x, s)]=0 .
$$

It follows that the limits $a, b$, of the integral in eq 8 , may as well be replaced by $-\infty,+\infty$, respectively.

The behavior of $G(s)$ in the interval where it does not vanish can best be illustrated by a number of simple examples.

Example 1. Let the basic set be $1, x, x^{2}, x^{3}$, let $x_{0}, x_{1}, x_{2}, x_{3}, x_{4}$ be five equally spaced values of $x$ with interval $h$, let $y_{i}=f\left(x_{i}\right)$ and let

$$
R[f(x)]=y_{4}-4 y_{3}+6 y_{2}-4 y_{1}+y_{0}=\Delta^{4} y_{0} .
$$

For this case

$$
\bar{g}(x, s)=(x-s)^{3} / 3 !, x>s,
$$

whence

$$
\begin{aligned}
& G(s)=R[\bar{g}(x, s)]=\frac{1}{3 !}\left[{\overline{\left(x_{4}-s\right)}}^{3}-4{\overline{\left(x_{3}-s\right)^{3}}}^{3}+\right. \\
& \left.6{\overline{\left(x_{2}-s\right)}}^{3}-4{\overline{\left(x_{1}-s\right)}}^{3}+{\overline{\left(x_{0}-s\right)}}^{3}\right],
\end{aligned}
$$

where the notation $\overline{(x-s)}^{3}$ means that

$$
\overline{(x-s)^{3}}=\left\{\begin{array}{cc}
(x-s)^{3} & \text { if } x \geqslant s, \\
0 & \text { if } x<s .
\end{array}\right.
$$

We now see at once that for

$$
\begin{array}{ll}
x_{4} s \leqslant<\infty & G(s)=0 \\
x_{3} s \leqslant<x_{4} & G(s)=\frac{1}{3 !}\left(x_{4}-s\right)^{3} \\
x_{2} \leqslant s<x_{3} & G(s)=\frac{1}{3 !}\left[\left(x_{4}-s\right)^{3}-4\left(x_{3}-s\right)^{3}\right] \\
x_{1} s \leqslant<x_{2} & G(s)=-\frac{1}{3 !}\left[-4\left(x_{1}-s\right)^{3}+\left(x_{0}-s\right)^{3}\right] \\
x_{0} s \leqslant<x_{1} & G(s)=-\frac{1}{3 !}\left[\left(x_{0}-s\right)^{3}\right] \\
-\infty<s<x_{0} & G(s)=0 .
\end{array}
$$


The graph of this function is shown in figure 1.

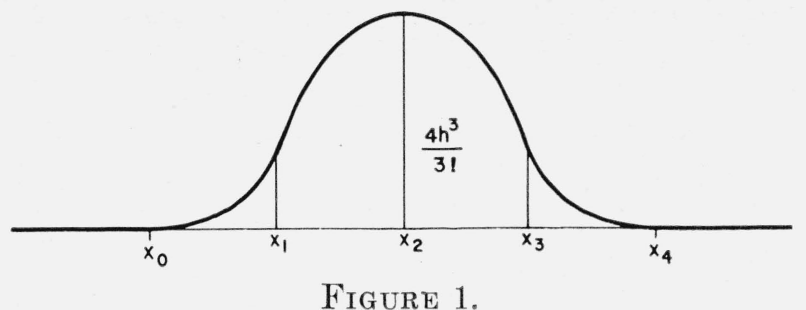

Thus it is seen that $G(s)$ consists of four ares of four different third-degree polynomial curves joined together continuously and with continuous first and second derivatives.

Since we started with $R[f(x)]=\Delta^{4} y_{0}$, we have shown that

$$
\Delta^{4} y_{0}=\int_{-\infty}^{\infty} f^{(4)}(s) G(s) d s,
$$

when $G(s)$ is the function just described.

Example 2. Let the basic set be $1, x, x^{2}, x^{3}, x^{4}$, let $x_{0}, x_{1}, x_{2}$ be three equally spaced points with interval $h$, and let

$$
R[f(x)]=y_{2}-y_{0}-\frac{h}{3}\left(y_{2}^{\prime}+4 y_{1}^{\prime}+y_{0}^{\prime}\right) \cdot
$$

(This is equivalent to Simpson's Rule).

For this case

$$
\bar{g}(x, s)=\overline{(x-s)^{4}} / 4 \text { ! }
$$

whence

$$
\begin{gathered}
G(s)=\frac{{\overline{\left(x_{2}-s\right)}}^{4}-\overline{\left(x_{0}-s\right)}}{4 !} \\
-\frac{h}{3 \times 3 !}\left[\overline{\left(x_{2}-s\right)^{3}}+4 \overline{\left(x_{1}-s\right)^{3}}+\overline{\left(x_{0}-s\right)^{3}}\right] .
\end{gathered}
$$

We have then:

$$
\begin{array}{ll}
x_{2} \leqslant s<\infty & G(s)=0 \\
x_{1} \leqslant s<x_{2} & G(s)=\frac{\left(x_{2}-s\right)^{4}}{4 !}-\frac{h\left(x_{2}-s\right)^{3}}{3 \times 3 !} \\
x_{0} \leqslant s<x_{1} & G(s)=\frac{\left(x_{0}-s\right)^{4}}{4 !}+\frac{h\left(x_{0}-s\right)^{3}}{3 \times 3 !} \\
-\infty<s<x_{0} & G(s)=0 .
\end{array}
$$

The graph of this function is shown in figure 2 . Here $G(s)$ consists of arcs of two fourth-degree polynomials joined together with third-degree contact.

Recalling the original definition of $R[f(x)]$ in this example we see that

$$
y_{2}-y_{0}-\frac{h}{3}\left(y_{2}^{\prime}+4 y_{1}^{\prime}+y_{0}^{\prime}\right)=\int_{-\infty}^{\infty} y^{(5)}(s) G(s) d s .
$$

The right-hand member of this equation is therefore a measure of the error of Simpson's rule in the special form chosen here.

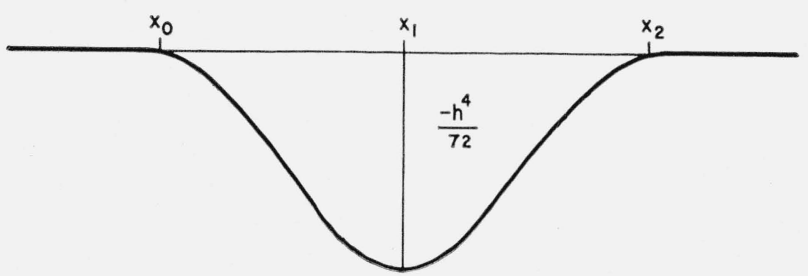

FIGURE 2.

Example 3. In the two preceding examples we have started with a known operator, $R$, and have derived from $R$ the function $G(s)$. Now that we see something of the character of $G(s)$ in the case of a polynomial basic set, it is clear that we could reverse the process-i. e., start with a $G(s)$ satisfying suitable conditions as to degree and continuity and derive from it the operator $R$. To illustrate this procedure we start with

$$
\begin{array}{ll}
G(s)=0 & \text { if } x_{1} \leqslant s, \\
G(s)=\left(x_{1}-s\right)^{3}\left(s-x_{0}\right)^{3} & \text { if } x_{0} \leqslant s<x_{1}, \\
G(s)=0 & \text { if } s<x_{0} .
\end{array}
$$

Here $G(s)$ is a polynomial of degree six, so that the basic set is $1, x, x^{2}, x^{3}, x^{4}, x^{5}, x^{6}$, the differential equation is $D^{7} u=0$, and

$$
\begin{aligned}
R[f(x)] & =\int_{-\infty}^{\infty} f^{(7)}(s) \overline{\left(x_{1}-s\right)^{3}} \overline{\left(s-x_{0}\right)^{3}} d s \\
& =\int_{x_{1}}^{x_{0}} f^{(7)}(s)\left(x_{1}-s\right)^{3}\left(s-x_{0}\right)^{3} d s .
\end{aligned}
$$

Now $G(s)=G^{\prime}(s)=G^{\prime \prime}(s)=0$ at $s=x_{0}$ and at $s=x_{1}$.

$$
\begin{array}{ll}
G^{\prime \prime \prime}\left(x_{0}\right)=6 h^{3} & G^{\prime \prime \prime}\left(x_{1}\right)=-6 h^{3} \\
G^{(4)}\left(x_{0}\right)=-72 h^{2} & G^{(4)}\left(x_{1}\right)=-72 h^{2} \\
G^{(5)}\left(x_{0}\right)=360 h & G^{(5)}\left(x_{1}\right)=-360 h \\
G^{(6)}\left(x_{0}\right)=-720 & G^{(6)}\left(x_{1}\right)=-720 .
\end{array}
$$

Integration by parts gives accordingly

$$
\begin{gathered}
R[f(x)]=6 h^{3}\left[f^{\prime \prime \prime}\left(x_{1}\right)+f^{\prime \prime \prime}\left(x_{0}\right)\right] \\
-72 h^{2}\left[f^{\prime \prime}\left(x_{1}\right)-f^{\prime \prime}\left(x_{0}\right)\right]+360 h\left[f^{\prime}\left(x_{1}\right)+f^{\prime}\left(x_{0}\right)\right] \\
-720\left[f\left(x_{1}\right)-f\left(x_{0}\right)\right] .
\end{gathered}
$$


Transposing and dividing by 720 we obtain the formula with remainder term

$$
\begin{aligned}
& f\left(x_{1}\right)-f\left(x_{0}\right)=\frac{h}{2}\left[f^{\prime}\left(x_{1}\right)+f^{\prime}\left(x_{0}\right)\right]-\frac{h^{2}}{10}\left[f^{\prime \prime}\left(x_{1}\right)-f^{\prime \prime}\left(x_{0}\right)\right]+ \\
& \frac{h^{3}}{120}\left[f^{\prime \prime \prime}\left(x_{1}\right)+f^{\prime \prime \prime}\left(x_{0}\right)\right]-\frac{1}{720} \int_{x_{0}}^{x_{1}} f^{(7)}(s)\left(x_{1}-s\right)^{3}\left(s-x_{0}\right)^{3} d s .
\end{aligned}
$$

This formula might be looked upon as the first few terms with remainder of a sort of two-point Taylor expansion. It can be used in successive approximations to secure a starting value in the step-by-step integration of a differential equation. Its virtue lies in the fact that while it requires only the first three derivatives it is as accurate as a Taylor's series using the first six derivatives.

This example illustrates that fact that $G(s)$ is a kind of generating function from which the particular operator $R$ associated with $G(s)$ can be obtained by carrying out the integration

$$
\int_{\infty}^{\infty} f^{(n+1)}(s) G(s) d s .
$$

It is this property which suggested the notation $G(s)$.

Example 4. In certain instances it may be easier to determine the contact properties by which the arcs composing the function $G(s)$ are joined and to construct $G(s)$ from these data rather than to compute $R\left[(x-s)^{n}\right]$ directly. An instance of this is the following: Let the basic set be $1, x, \ldots, x^{n}$, and let $R[f(x)]$ represent the difference between $f(x)$ and the best $n$-th degree polynomial approximation by Least Squares for the interval $(-1,1)$. We shall suppose that $x$ is in this interval. A brief examination will show that $G(s)$ is of degree $n$ in $x$, of degree $2 n+1$ in $s$, vanishes at $s=1$ to the same order as $(1-s)^{n+1}$, vanishes at $s=-1$ to the same order as $(1+s)^{n+1}$, and if $G_{\mathrm{R}}$ and $G_{\mathrm{L}}$ are the polynomials to the right and left respectively of $s=x$, then

$$
G_{L}=\frac{(x-s)^{n}}{n !}+G_{R}
$$

These data determine $G_{R}$ and $G_{L}$ completely and after considerable algebraic reduction it is found that

$$
\begin{gathered}
G(s)=\frac{1-s^{n+1}}{2^{2 n+1} n !}\left[\sum_{k=0}^{n} \frac{1}{\left.2^{k} k ! \frac{d^{k}}{d x^{k}}\left\{(x+1)^{n}(x-1)^{k}\right\}(1+s)^{k}\right],}\right. \\
\text { for } x<s<1 .
\end{gathered}
$$

The foregoing examples furnish an idea of the character of $(G s)$ when the basic set consists of polynomials. Similarly when the basic set is trigonometric we would expect to find that $G(s)$ is composed of trigonometric arcs welded together; for the exponential case, $G(s)$ would be made up of exponential arcs, etc.

\section{Computation of the Error}

As has been shown, the remainder is of the form

$$
R[f(x)]=\int_{-\infty}^{\infty} L(f(s)) G(s) d s .
$$

If $a \leqq s \leqq b$ is the interval in which $G(s)$ is not identically zero, we have

in which

$$
|R[f(x)]|<M K(b-a),
$$

$$
\begin{gathered}
M=\max |L(f(s))| \text { in } a \leqslant s \leqslant b, \\
K=\max |G(s)| \text { in } a \leqslant s \leqslant b .
\end{gathered}
$$

The inequality 10 provides a bound for the magnitude of the error in all cases.

In many particular problems this result can be considerably improved. For example, if $G(s)$ does not change sign in the interval $(a, b)$ we may apply the law of the mean for integrals and write

$$
R[f(x)]=L(f(\xi)) \int_{-\infty}^{\infty} G(s) d s,
$$

where $a<\xi<b$. Note that this is the case for examples 1, 2, and 3 of section IX. Since in those examples we have the explicit expressions for the several ares of which $G(s)$ is composed, there is no difficulty in performing the integration over each arc and thus we can obtain the actual value of the integral

$$
\int_{-\infty}^{\infty} G(s) d s .
$$

However we now show that the value of the above integral may be obtained from the operator $R$ without making any use of the explicit form of $G(s)$. Let $w(x)$ be any solution of the differential equation

$$
L(w)=1,
$$

and replace $f(x)$ in eq 8 by $w(x)$. The result is

$$
R[w(x)]=\int_{-\infty}^{\infty} G(s) d s .
$$


By using this result in eq 11 we have

$$
R[f(x)]=L(f(\xi)) R[w(x)],
$$

provided $G(s)$ does not change sign.

\section{Polynomial Approximation}

For the polynomial case where

$$
L(u)=\frac{d^{n+1} u}{d x^{n+1}}=0,
$$

a particular solution of $L(w)=1$ is

$$
w(x)=x^{n+1} /(n+1) ! .
$$

Hence we have the theorem:

if, (a) the basic set is $1, x, \ldots, x^{n}$, and

(b) the function $G(s)$ does not change sign, then the error is

$$
R[f(x)]=\frac{f^{(n+1)}(\xi)}{(n+1) !} R\left[x^{n+1}\right],
$$

where $a<\xi<b$.

This result contains many well-known expressions for the error as special cases. For example, in the case of polynomial interpolation eq 13 reduces to

$$
R[f(x)]=\frac{f^{(n+1)}(\xi)}{(n+1) !}\left(x-x_{0}\right)\left(x-x_{1}\right) \ldots\left(x-x_{n}\right) .
$$

In the type of interpolation where both $f(x)$ and $f^{\prime}(x)$ are given by the approximating polynomial at the given points, eq 13 becomes

$$
R[f(x)]=\frac{f^{(2 n+2)}(\xi)}{(2 n+2) !}\left(x-x_{0}\right)^{2}\left(x-x_{1}\right)^{2} \ldots\left(x-x_{n}\right)^{2} .
$$

In the case of Newton-Cotes quadrature formulas, eq 13 also gives the same results as those obtained by the use of Bernoulli polynomials.

Example 1. In the case of Newton's "three eighth's rule" in the form

$$
y_{3}-y_{0}-\frac{3 h}{8}\left(y_{3}^{\prime}+3 y_{2}^{\prime}+3 y_{1}^{\prime}+y_{0}^{\prime}\right)=R(y),
$$

the basic set is $1, x, x^{2}, x^{3}, x^{4}$, and the points are $x_{0}, x_{0}+h, x_{0}+2 h, x_{0}+3 h$.

There is no loss in generality if we take $x_{c}=0$. since the formula is independent of any particular location on the $x$-axis. Then

$$
R\left[x^{5}\right]=\left\{3^{5}-\frac{15}{8}\left(3^{4}+3 \times 2^{4}+3 \times 1^{4}+0^{4}\right)\right\} h^{5}=-9 h^{5} / 2,
$$

and the error turns out to be

$$
|R[y]|=\frac{y^{(5)}}{5 !} \cdot \frac{9 h^{5}}{2}=\frac{3 h^{5} y^{(5)}}{80} .
$$

Here, and elsewhere below, the argument of the derivative in the remainder has been suppressed.

The theorem also applies to a wide variety of approximate formulas that are not commonly treated in texts on interpolation and numerical integration, and to which the customary methods for obtaining the error do not directly apply.

A few such formulas, together with error terms, are listed below.

(1) $y_{n}-y_{n-1}=\frac{h}{2}\left(y_{n}^{\prime}+y_{n-1}^{\prime}\right)-\frac{h^{2}}{12}\left(y_{n}^{\prime \prime}-y_{n-1}^{\prime \prime}\right)+\frac{h^{5} y^{(5)}}{720}$.

$$
\begin{gathered}
y_{n}-y_{n-1}=\frac{h}{2}\left(y_{n}^{\prime}+y_{n-1}^{\prime}\right)-\frac{h^{2}}{10}\left(y_{n}^{\prime \prime}-y_{n-1}^{\prime \prime}\right) \\
+\frac{h^{3}}{120}\left(y_{n}^{\prime \prime \prime}-y_{n-1}^{\prime \prime \prime}\right)-\frac{h^{7} y^{(7)}}{100800} .
\end{gathered}
$$

(3) $y_{n+1}=y_{n}+\frac{h}{24}\left(7 y_{n+1}^{\prime}+16 y_{n}^{\prime}+y_{n-1}^{\prime}\right)+\frac{h^{2} y_{n}^{\prime \prime}}{4}-\frac{h^{5} y^{(5)}}{180}$.

$$
\begin{gathered}
y_{n+2}=y_{n}+\frac{2 h}{3}\left(5 y_{n+1}^{\prime}-y_{n}^{\prime}-y_{n-1}^{\prime}\right)-2 h^{2} y_{n}^{\prime \prime} \\
+\frac{7 h^{5} y^{(5)}}{45} .
\end{gathered}
$$

$$
\begin{aligned}
y_{n+1}= & y_{n-1}+{ }_{15}^{h}\left(7 y_{n+1}^{\prime}+16 y_{n}^{\prime}+7 y_{n-1}^{\prime}\right) \\
& -\frac{h^{2}}{15}\left(y_{n+1}^{\prime \prime}-y_{n-1}^{\prime \prime}\right)+\frac{h^{7} y^{(7)}}{4725} .
\end{aligned}
$$

(6) $y_{n+1}-2 y_{n}+y_{n-1}=\frac{3 h}{8}\left(y_{n+1}^{\prime}-y_{n-1}^{\prime}\right)$

$$
-\frac{h^{2}}{24}\left(y_{n+1}^{\prime \prime}-8 y_{n}^{\prime \prime}+y_{n-1}^{\prime \prime \prime}\right)+\frac{h^{8} y^{(8)}}{60480} \text {. }
$$

(7)

$$
\begin{aligned}
& y_{n+1}=y_{n-1}+\frac{h}{105}\left(41 y_{n+1}^{\prime}+128 y_{n}^{\prime}+41 y_{n-1}^{\prime}\right) \\
& -\frac{2 h^{2}}{35}\left(y_{n+1}^{\prime \prime}-y_{n-1}^{\prime \prime}\right)+\frac{h^{3}}{315}\left(y_{n+1}^{\prime \prime \prime}+16 y_{n}^{\prime \prime \prime}+y_{n-1}^{\prime \prime \prime}\right) \\
& -\frac{h^{11} y^{(11)}}{130,977,000} .
\end{aligned}
$$

These formulas have applications in the numerical solution of differential equations. 


\section{Future Investigations}

So far, the possibilities of securing usable remainder terms by the methods of this paper have not been fully explored. It is, for example, unknown whether these methods will give practically useful forms of the remainder for the case of polynomial approximation by Least Squares, for the case of Fourier series, and more general Fourier type expansions. These cases have, of course, been extensively studied by Dunham Jackson and others by using a very different line of attack.

Another matter deserving attention is the dis- covery of general theorems by which it can be determined for whole classes of operators whether or not the associated function $G(s)$ changes sign. For we have seen that when we know that $G(s)$ does not change sign the evaluation of the magnitude of the error is considerably simplified. On the other hand, if it is necessary to compute $G(s)$ in order to determine whether or not $G(s)$ changes sign, the benefit of this simplification is considerably reduced. Except in a few special cases such as polynomial interpolation, no such general theorems are known.

Los Angeles, February 25, 1949. 